\title{
Illusory contours from inducers defined solely by spatiotemporal correlation
}

\author{
K. PRAZDNY \\ Artificial Intelligence Laboratory, Santa Clara, California
}

\begin{abstract}
Vivid and perceptually salient subjective contours are perceived when inducing (two-dimensional) objects that move and change form so as to "simulate" the presence of an occluding shape are defined solely by (the differences in) temporal correlation in random-dot cinematograms. These effects suggest that subjective contours are due to mechanisms not directly tied to a single source of sensory information, and challenge accounts based on low-level brightness-domain computations.
\end{abstract}

The percept of subjective (or illusory) contours (Kanisza, 1979; Schumman, 1904) refers to three different, although possibly related, observations-edges enclosing an occluding surface (which has brightness usually markedly different from that of the surround) are seen in the absence of physical stimuli usually associated with them. To be explained are, thus, the (illusory) stratification (perception of one surface in front of another), the (illusory) brightness difference, and the phenomenally present but physically nonexistent contours.

The hypotheses relevant to these phenomena can be divided into two broad classes. The bottom-up explanations are based on physiological data and maintain that the subjective contour (SC) is a necessary consequence of the working of the underlying physiological "wetware" (e.g., low-level feature detectors or lateral inhibition units). Common to these accounts is the belief that some form of simultaneous brightness contrast is the direct cause of the perception of the illusory contour and the occlusion. This general approach is perhaps best exemplified by the work of Frisby and Clatworthy (1975) and Jory and Day (1979), who claim that a region of enhanced brightness (induced by lateral inhibition), once formed, spreads out to fill the shape outlined by small features such as line ends and points. The plausibility of these hypotheses has been weakened by recent findings (Cohen \& Grossberg, 1984; Prazdny, 1983; Shapley \& Gordon, 1985) that subjective contours occur also in displays in which the inducing elements have opposite contrast. This fact poses difficulties not only for theories based on the simple simultaneous-brightness contrast-like phenomena (e.g., Brigner \& Gallagher, 1974; Frisby \& Clatworthy, 1975; Kennedy \& Lee, 1976), but also for other (bottom-up) explanations based on spatial frequency filtering (e.g., Ginsburg, 1975). Recently, a nonlinear brightness-domain spatial filter was proposed which can (in principle, at least) account for the perception of sub-

The author's mailing address is Machine Perception Group, Artificial Intelligence Laboratory, FMC, 1185 Coleman Ave., Box 580, Santa Clara, CA 95052. jective contours in the opposite-contrast displays (Cohen \& Grossberg, 1984; Shapley \& Gordon, 1985). The filter (which is essentially a logical "exclusive or" function in the contrast domain) is sensitive to the orientation and amount of local contrast but not to its direction.

Other, top-down or cognitive, accounts (Gregory, 1972) regard SC as being only suggested by sensory information. In these theories, certain stimulus features elicit a conceptual structure (a model or a prototype), which is then tested against available evidence and (when confirmed) literally imposed on the sensory data.

Here we report that subjective figures are perceived in displays in which the (moving) inducing forms are defined solely by temporal correlation. The stimuli were random-dot cinematograms: a sequence of static randomdot distributions displayed in rapid succession as a motion picture. Each static snapshot (movie frame) contains only a set of randomly placed black dots and does not contain any information at all. Inducing figures are defined only by temporal correlation (or the lack of it) and are bounded by kinetic edges: places of sharp discontinuity in motion direction and/or magnitude. In contrast to previous work by Bradley and Lee (1982) and Kellman and Cohen (1984), which demonstrated kinetic subjective contours with luminance-domain stimuli, in these situations the perception of subjective contour is obtained even though no static brightness information is present to specify either the subjective contour or even, in fact, the inducing elements. The occurrence of illusory contours in such displays is important for at least two reasons: (1) It shows that the origin of inducing forms is not a relevant variable (and thus that, e.g., the Cohen/Grossberg operators do not capture the essence of the phenomenon), and (2) it suggests that the relevant processes are located at or beyond the level at which the structure is computed from motion, and also indicates that brightness domain effects are not a necessary ingredient.

Current psychophysical evidence supports the view that the human visual system contains (at least) two different mechanisms involved in the process of motion detection and measurement (Braddick, 1974). In general, there are two possible ways in which a visual system can detect mo- 
tion. Motion measurements can be based directly on the (local) changes in some function of (retinal) brightness values (Hassenstein \& Reichardt, 1956; Limb \& Murphy, 1975; Marr \& Ullman, 1981), or, alternatively, features such as edges, blobs, corners, and even whole regions may first be identified and then "tracked" (i.e., their identity maintained) over time (Anstis, 1980; Ullman, 1979). The latter kind of motion detector is, in principle, not bound to one information source; that is, such a detector can detect motion of brightness as well as, for example, stereoscopic edges (Julesz \& Payne, 1968).

Logically, at least, it is possible to use the "short-range" (brightness domain) motion detectors (which can process displacements of up to about $20^{\prime}$ ) to define kinetic forms (by discontinuities in motion), which can then in turn be used as figures in the classical apparent-motion situation (Petersik, Hicks, \& Pantle, 1978; Sperling, 1976). This is the stimulus configuration used in our experiments. Consider, for example, Figure 1, which shows three snapshots from a movie sequence which, when displayed in rapid succession, elicits the perception of two black rectangles rotating under an illusory triangle. All three components of the phenomenon (illusory contour, brightness enhancement, and apparent stratification) can be seen in these displays even though no single movie frame contains the static subjective contour. Kinetic shapes are easily obtained (using a computer) from these black/white "templates" by replacing all dark regions with dynamic (snow) noise (i.e., each movie frame contains a different distribution of random dots within the region) and replacing the surround with the static random-dot noise (i.e., the same background random-dot field is present in all frames). Because the position and orientation of these kinetic shapes varies from snapshot to snapshot, one perceives them as moving. Observe that in this situation it is the information provided by the low-level, short-range motion detector which is integrated over space to define "edges" for the higher level processing responsible for the perception of motion (rotation) of the kinetic shapes. ${ }^{1}$

\section{Method}

The black/white random-dot cinematograms [ $p$ (white) $=p$ (black) $=0.5]$ were prepared and displayed on a Symbolics 3670 Lisp machine with high resolution noninterlaced display (refresh rate $60 \mathrm{~Hz}$ ) and fast (P4) phosphor. The display was a square of $290 \times 290$ pixels and was viewed from a distance of about 20 in. (i.e., 1 pixel $=2.15^{\prime}$ ). The subjective figure subtended about $2.25^{\circ}$ of visual arc. In our experiments, each snapshot of the original black/white display was defined by a sequence of up to five movie frames. The frame duration was $33 \mathrm{msec}$. At least two frames were necessary in each sequence to define the kinetic forms by producing discontinuities in motion. The display consisted of (up to) $\mathbf{3 0}$ snapshots (sequences). Stimulus onset asynchrony (SOA) between the snapshots was controlled by varying the number of frames in each (sub)sequence (minimum 2, maximum 5).

\section{RESULTS}

Twenty subjects viewed the displays, and all (without exception) could correctly identify the subjective figure
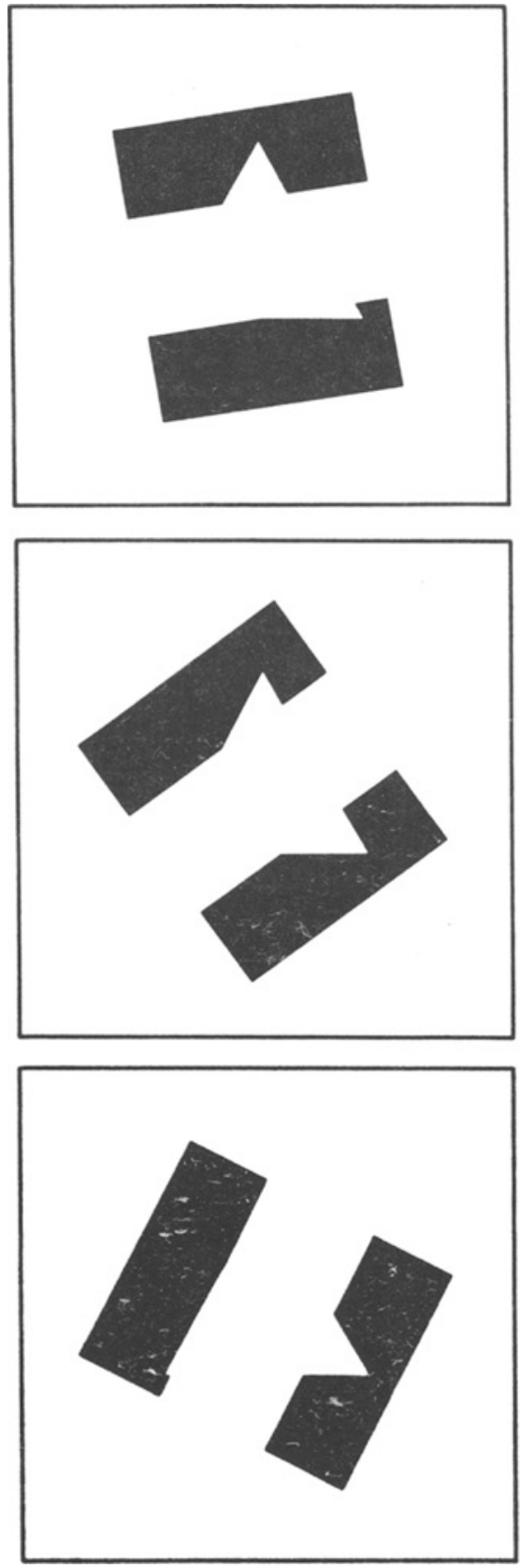

Figure 1. Three frames of a computer-generated movie picture sequence "simulating" the occlusion of two rotating rectangles by a "nonexistent" stationary opaque shape (triangle here). Subjective contour in its full glory is perceived when the inducers are seen to rotate (and change shape as the "protrusions walk over" the rectangles), even though no single static picture frame contains a configuration that generates the stbjective contours. Purely kinetic moving inducing objects (with no reality in the brightness domain) can be created by filling the dark regions with dynamic (smow) and the white beckground with static random noise. The kinetic forms are created by discontinuities in motion (generated by the activity of an intencify based, low tevel, short-range motion detector); thelr motion is detected by a bigher level mechanism independent of huminance-domsin proceseing. Such kinetic stimuli generote perceptually very salien sebjective contour effects. 
(an equilateral triangle, a right-angle triangle, a rectangle, or a parallelogram). All viewers reported the perception of subjective figures together with apparent stratification and the presence of faint illusory edges (no subject reported any brightness effects). ${ }^{2}$ The SOA variations (67 to $167 \mathrm{msec}$ ) had no significant effect on the perception of subjective contour. The ISI between the two consecutive snapshots (sequences) was varied between 0 and $67 \mathrm{msec}$, but again this had no effect on the perceptions (ISI between the frames in each sequence was always 0 ). Random dots "inside" the inducing objects can also move in a coherent fashion (e.g., rigidly to the right) instead of randomly. Strong subjective contours are also perceived in this situation.

When the kinetic objects were defined by stationary random-dot noise and the background moved randomly (dynamic "snow" noise), no subjective contour effects could be obtained. This is an interesting and important finding, since presumably the same information (motion discontinuities) is present in this case as is when subjective contours are seen. In this situation, the static random dots are truly static, that is, they retain their image position while only the overall configuration rotates. Thus, the perception is slightly paradoxical, perhaps equivalent to that obtained when seeing a stationary texture through a rotating window or a hole (although such literal percepts were not reported in our experiments). It seems that some higher level organizational factors that may be responsible for such literal percepts inhibit the perception of subjective figures in this case. Prazdny (1985) reported that static or purely translating inducers defined by motion discontinuities in the kinetic domain do not result in the perception of subjective contours. Why does rotation but not translation produce subjective figures? The displays used in those experiments were random dots correlated (across time) inside the inducers but uncorrelated in the background (dynamic random noise). The motion of inducers in these displays is not associated with the accretion/deletion effects that usually accompany the perception of depth stratification of moving objects. Rotating inducers sequentially "expose" or define different pieces of contours of the "subjective" figure. Simply translating inducers define only one and the same part of the (illusory) object. As a speculation, it may be that in the absence of the accretion/deletion effects, a sequential exposure of different parts of a subjective figure is needed to obtain the illusion (similar to the anarthoscopic situation). More research is needed on this interesting issue.

Another interesting situation is obtained when the background moves coherently in one direction and the inside of the inducers is either stationary or moves in another direction or with different speed. In these displays, one can always identify the correct shape of the subjective figure. However, the perceived structure is sometimes severely distorted. This is due to the fact that the moving background "captures" (i.e., carries with it) the "subjective" edge. For example, when the background moves rigidly to the left, it displaces the phenomenal position of the edges (currently being "exposed" by the moving inducers ) to the left so that some time later they are "incorrectly" combined with another part of the subjective figure that is being "exposed" at that time. ${ }^{3}$ Similar "capture" effects were reported recently by Spillman and Redies (1981), who observed that when a transparent sheet of random dots is moved in front of Ehrenstein figures, the illusory patches seem to move in the same direction and out of the inducing area while maintaining their overall organization. In this case, however, no distortion takes place because the Ehrenstein figures are all defined simultaneously!

\section{CONCLUSION}

Subjective contours can be perceived in displays which, when viewed as a sequence of static pictures in isolation, do not produce the illusion; they are especially strong when the inducers themselves move and change form so as to "simulate" stationary, opaque occluding figures (Bradley \& Lee, 1982; Kellman \& Cohen, 1984). Our experiments show that the same vivid and perceptually salient phenomena occur readily when the (moving) inducers are defined solely by differences in temporal correlation, that is, in the absence of any brightness-domain information. These findings are intriguing because they suggest that the locus of the subjective contour phenomena may be beyond operations exemplified by primitive feature extraction. Subjective contours can also easily be seen when one of the inducers in Figure 1 is defined in the brightness domain (a black form against the background of dynamic noise) while the other exists only in the kinetic domain (static noise against the background of random motions). This suggests that the phenomenon could take place at cortical regions where signals from the two domains are brought together. Similar findings that show that the kinetic depth effect (Wallach \& O'Connell, 1953) occurs when moving objects are defined solely by motion or disparity discontinuities (Prazdny, 1986) and recent neurophysiological results that show that an illusory contour excites area 18 but not area 17 neurons (Von der Heydt, Peterhans, \& Baumgartner, 1984) suggest that the phenomenon is not a peripheral one but a more central event reflecting interpolative (i.e., not based on direct evidence) capabilities of the visual system. ${ }^{4}$

\section{REFERENCES}

ANsTis, S. M. (1980). The perception of apparent movement. Philosophical Transactions of the Royal Society, London, B-290, 153-168.

Braddick, O. J. (1974). A short range process in apparent motion. Vision Research, 14, 519-528.

Bradley, D., \& LeE, K. (1982). Animated subjective contours. Perception \& Psychophysics, 32, 393-395.

Brigner, W. L., \& Gallagher, M. B. (1974). Subjective contour: Apparent depth or simultaneous brightness contrast? Perceptual \& Motor Skills, 38, 1047-1053.

Cohen, M A., \& Grossberg, S. (1984). Neural dynamics of brightness perception. Perception \& Psychophysics, 36, 428-456. 
Frisby, J. P., \& Clatworthy, J. L. (1975). Illusory contours: Curious cases of simultaneous brightness contrast. Perception, 4, 349-357

GinsBURG, A. P. (1975). Is the illusory triangle physical or imaginary? Nature, 257, 219-220.

Gregory, R. (1972). Cognitive contours. Nature, 238, 51-52.

Hassenstein, B., \& Reichardt, W. (1956). Systemtheoretische Analyse der Zeit-, Reihenfolgen- und Vorzeichenauswertung bei der Bewegungsperzeption der Russelkafers. Chlorophanus Zeitschrifi für Naturforschung, IIb, 513-524.

JoRY, M. K., \& DAY, R. H. (1979). The relationship between brightness contrast and illusory contours. Perception, 8, 3-9.

Julesz, B., \& PAYNe, R. A. (1968). Differences between monocular and binocular stroboscopic movement perception. Vision Research, 8, 433-444.

Kanisza, G. (1979). Organization in vision. New York: Praeger.

Kellman, P. J., CoheN, M. H. (1984). Kinetic subjective contours Perception \& Psychophysics, 35, 237-244.

KENNEDY, J. M., LEE, H. (1976). A figure density hypothesis and illusory contour brightness. Perception, 5, 387-392.

LIMB, J. O., \& MURPHY, J. A. (1975). Estimating velocity of moving images in television signals. Computer Vision, Graphics \& Image Processing, 4, 311-327.

MaRR, D., Ullman, S. (1981). Directional selectivity and its use in early vision. Proceedings of the Royal Society, London, B-211, 151-180.

Petersik, J. T., Hicks, K. I., \& Pantle, A. J. (1978). Apparent movement of successively generated subjective figures. Perception, 7 , 371-383.

Prazdny, K. (1983). Illusory contours are not caused by simultaneous brightness contrast. Perception \& Psychophysics, 34, 403-404.

Prazdny, K. (1985). On the nature of inducing forms generating perceptions of illusory contours. Perception \& Psychophysics, 37, 237-242.

Prazdny, K. (1986). Kinetic depth effect from motion and disparity discontinuities. Manuscript submitted for publication.

Schumman, F. (1904). Einige Beobachtungen über die Zusammenfassung von Gesichtdrucken zu Einheiten. Psychologische Studien, 1, 1-32.

ShAPLEY, R., \& GoRdON, J. (1985). Nonlinearity in the perception of form. Perception \& Psychophysics, 37, 84-88.

SPERLING, G. (1976). Movement perception in computer driven visual displays. Behavior Research Methods, Instruments, \& Computers, 8 , 144-151.
Spillman, L., \& Redies, C. (1981). Random-dot motion displaces Ehrenstein illusion. Perception, 10, 411-415.

UlLMAN, S. (1979). The interpretation of visual motion. Cambridge, MA: MIT Press.

Von der Heydt, R., Peterhans, E., \& Baumgartner, G. (1984). Illusory contours and cortical neuron responses. Science, 224, 1260-1262.

WALLACH, H., \& O'CoNnell, D. N. (1953). The kinetic depth effect. Journal of Experimental Psychology, 45, 205-217.

\section{NOTES}

1. It can perhaps be argued that it is not motion but again luminance discontinuities that are responsible for the definition of the inducing elements in these displays. This is because there is a difference in the distribution of black/white texture elements over time between the "dynamic" and the "static" regions. At a global level, the average brightness of the two regions is the same, but at a more microscopic (point) level it is not. At any particular point in the "static" region, the point is either black or white, whereas in the "dynamic" regions a point changes brightness over time. One should note, however, that moving the eyes or the head does not change the perception of subjective figures in these displays, even though in this situation every point changes its brightness due to retinal motions.

2. Weak subjective figures can also be seen when the illusory figure moves and the inducing forms are stationary. Only 2 of the 20 observers reported illusory edges in this situation although everyone reported apparent stratification and identified the illusory figure correctly (similar asymmetry has also been reported by Bradley \& Lee, 1982, and Kellman \& Cohen, 1984, in the brightness domain).

3. The "capture" effects diminish when the center of the subjective figure is fixated.

4. The fact that illusory edges are "faint" under some conditions and only the depth stratification effect is strong could be important, since it may be taken to argue against "cognitive" style of explanation. It is possible that different aspects of the percepts are attributable to different processes. The perceptual system may operate to demark and integrate stimulation gradients (i.e., discontinuities in brightness, depth, motion, texture, etc.) and the mechanisms responsible for subjective contours may exist at all levels (and within all channels) of the visual system.

(Manuscript received December 9, 1985; revision accepted for publication February 26, 1986.) 